\title{
Articles
}

\section{D-QSAR Study on the Influence of Alrylamino (R) Substituents on Herbicidal Activity of Thiourea Analogues}

\author{
Min-Gyu Soung, Kwan-Yong Park, and Nack-Do Sung* \\ Division of Applied Biology and Chemistry, College of Agriculture and Life Science, Chungnam National University, \\ Daejeon 305-784, Korea.*E-mail: ndsung15@hanmail.net \\ Received December 2, 2009, Accepted January 11, 2010
}

\begin{abstract}
Influences of alrylamino (R) substituents on the herbicidal activity $\left(\mathrm{pI}_{50}\right)$ of 1-(4-chloro-2-fluoro-5-propargyloxypheny)3-(R)-thiourea analogues $(\mathbf{1} \sim \mathbf{3 5})$ against the barnyard grass (Echinochloa crusgalli) in the pre-emergence step were discussed quantitatively using comparative molecular field analysis (CoMFA) and comparative molecular similarity indices analysis (CoMSIA) as the three dimensional quantitative structure-activity relationship (3D-QSAR) method. The statistically most satisfactory CoMFA models for the herbicidal activity against the barnyard grass had the better predictability $\left(\mathrm{r}_{\text {cv. }}^{2}\right)$ and correlativity $\left(\mathrm{r}_{\text {ncv. }}^{2}\right)$ than those of CoMSIA models. The optimized CoMFA model 1 $\left(r_{\text {cv. }}^{2}=0.531 \& r_{\text {ncv. }}^{2}=0.931\right)$ with the sensitivity to the perturbation $\left(\mathrm{d}_{\mathrm{q}}{ }^{2} / \mathrm{dr}_{\mathrm{yy}}^{2}=1.081\right)$ and the prediction $\left(\mathrm{q}^{2}=0.475\right)$ produced by a progressive scrambling analyses were not dependent on chance correlation. And statistical qualities with the atom based fit alignment (AF) were slightly higher than those of the field fit alignment (FF). According to the optimized CoMFA model 1, the contribution ratio (\%) of the steric field (76.9\%) on the herbicidal activity of the Thioureas was three-fold higher than that of the electrostatic field $(20.1 \%)$ and the hydrophobic field $(3.0 \%)$ had the least influence. A steric favor group is on the vicinity of the nitrogen atom in alrylamino (R) substituent, and a steric disfavor group is on the outer side of alrylamino (R) substituent. Thus, as the size of alrylamino (R) substituent increases, so does the herbicidal activity of the substituent.
\end{abstract}

Key Words: CoMSIA \& CoMFA analysis, 1-(4-Chloro-2-fluoro-5-propargyloxypheny)-3-(R)-thioureas, Herbicidal activity, Barnyard grass (Echinochlor crusgalli)

\section{Introduction}

Since oxadiazon was discovered as an herbicidal compound of the cyclic imides (CyI) family, many other CyI family derivatives have been developed. And their herbicidal modes of action in the inhibition of protoporphyrinogen IX oxidase (protox) activity have been studied widely. ${ }^{1}$ To develop new CyI family peroxidizing herbicides, studies on molecular similarity between herbicidal compounds and the protoporphyrinogen IX (protogen), which is a substrate molecule of protox (EC. 1.3.3.4) enzyme, have been reviewed. ${ }^{2,3}$

Most compounds such as CyI family peroxidizing herbicides, S-275, S-23121, Sulfentrazone, and Carfentrazone ethyl include the same structure as the $N$-aryl group. Recently, studies on the peroxidizing herbicidal activity of thiourea family instead of the cyclic moiety were examined. ${ }^{4}$ Also, the role of substituent on the benzene ring specifically attracted attention and the introduction of hetero cyclic compounds that are condensed into a benzene ring at the meta- and para-position has been tried. ${ }^{5}$ For peroxidizing herbicides, the CyI and the diphenyl ether family compounds are widely used. It is expected that these herbicides are selectively toxic between animals and plants because the chlorophyll in their plant cells is an active site. ${ }^{6}$ The recent quantitative structure-activity relationships (QSARs) on the herbicidal activity of 2-alkylphenyl-3-phenylthioisoindoline-1-one, ${ }^{7}$ 2-(4-chloro-5-(2-chloroallyloxy)-2-fluorophenyl)- 3-thio-alkoxy-2,3,4,5,6,7-hexahydro-isoindol-1-ones, ${ }^{8}$ and 5benzofuryl-2-[1-(alkyloxyimino)-alkyl]-3-hydroxycyclohex2 -en-1-ones ${ }^{9}$ as the Cyl family compounds were studied extensively. From these studies, it was reported that the 2-fluoro4-chloro-5-alkoxyanilino substituent was effective on herbicidal activity. ${ }^{1,8}$

In the present study, to understand the structural basis for the herbicidal activity and to guide the design of more potent compounds, the influences of alrylamino (R) substituents on the herbicidal activity $\left(\mathrm{pI}_{50}\right)$ of new 1-(4-chloro-2-fluoro-5propargyloxypheny)-3-(R)-thiourea analogues $(\mathbf{1} \sim \mathbf{3 5})$ against the barnyard grass (Echinochloa crusgalli) in the pre-emergence step were discussed quantitatively using the 3D-QSAR (CoMSIA \& CoMFA) methods. ${ }^{10}$ Particulaly, the CoMFA method is not only one of the most used 3D-QSAR methods, but has also been applied to a number of different classes of compounds. ${ }^{11}$

\section{Materials and Methods}

Molecular modeling. The author's experimental data ${ }^{12}$ were taken from the herbicidal activity $\left(O b s . \mathrm{pI}_{50}\right)$ of 1-(4-chloro-2fluoro-5-propargyloxypheny)-3-(R)-thiourea analogues (herein after referred to as the Thioureas) (R: alkyl $=\mathbf{1} \sim \mathbf{1 8}$ and aryl $=$ $19 \sim 35$ ) observed (Table 1) against the barnyard grass (Echinochloa crusgalli) (hereinafter referred to as the EC) in the 
Table 1. Herbicidal activity $\left(\mathrm{pI}_{50}\right)$ of the Thioureas against the barnyard grass and predicted activity by the optimized CoMFA model 1 for training set

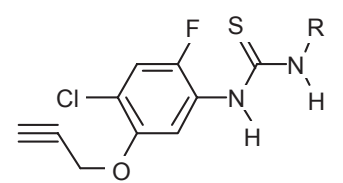

\begin{tabular}{|c|c|c|c|c|}
\hline \multirow{2}{*}{ No. } & \multirow{2}{*}{ Substituents (R) } & \multicolumn{3}{|c|}{$\mathrm{pI}_{50}$} \\
\hline & & Obs. ${ }^{a}$ & Pred. $^{b}$ & $\Delta \mathrm{pI}_{50}{ }^{\mathrm{c}}$ \\
\hline 1 & $n$-propyl & 3.65 & 3.71 & -0.06 \\
\hline 2 & $n$-butyl & 3.32 & 3.45 & -0.13 \\
\hline 3 & $n$-pentyl & 3.28 & 3.33 & -0.05 \\
\hline 4 & $n$-hexyl & 3.20 & 3.31 & -0.11 \\
\hline 5 & $n$-octyl & 3.34 & 3.30 & 0.04 \\
\hline 6 & allyl & 4.71 & 4.63 & 0.08 \\
\hline 7 & iso-propyl & 4.22 & 4.29 & -0.07 \\
\hline 8 & cy-propyl & 3.73 & 3.68 & 0.05 \\
\hline 9 & cy-butyl & 3.98 & 4.00 & -0.02 \\
\hline 10 & cy-pentyl & 3.85 & 3.93 & -0.08 \\
\hline 12 & cy-heptyl & 3.56 & 3.41 & 0.15 \\
\hline 13 & cy-octyl & 3.66 & 3.69 & -0.03 \\
\hline 14 & di-ethyl & 4.00 & 3.95 & 0.05 \\
\hline 16 & di- $n$-propyl & 3.23 & 3.13 & 0.10 \\
\hline 18 & di-allyl & 4.39 & 4.36 & 0.03 \\
\hline 21 & 4-fluorophenyl & 4.00 & 3.91 & 0.09 \\
\hline 22 & 4-methoxyphenyl & 3.56 & 3.65 & -0.09 \\
\hline 23 & 4-bromophenyl & 3.74 & 3.62 & 0.12 \\
\hline 24 & 4-hydroxyphenyl & 3.38 & 3.39 & -0.01 \\
\hline 25 & 4-nitrophenyl & 3.06 & 3.03 & 0.03 \\
\hline 26 & 3-chlorophenyl & 3.33 & 3.30 & 0.03 \\
\hline 27 & 3-bromophenyl & 3.25 & 3.23 & 0.02 \\
\hline 28 & 3-hydroxyphenyl & 2.91 & 3.48 & 0.02 \\
\hline 30 & 3-methoxyphenyl & 3.62 & 3.48 & 0.14 \\
\hline 31 & 3-tolyl & 2.57 & 2.83 & -0.26 \\
\hline 32 & 3-Cl, 4-F-phenyl & 3.56 & 3.69 & -0.13 \\
\hline 33 & 3,5-difluorophenyl & 3.35 & 3.52 & -0.17 \\
\hline 34 & 3,5-dimethylphenyl & 3.12 & 2.85 & 0.27 \\
\hline
\end{tabular}

${ }^{a}$ Observed herbicidal activity; ${ }^{b}$ predicted activity by the optimized CoMFA model $1 ;^{c}$ different between the observed and the predicted activity.

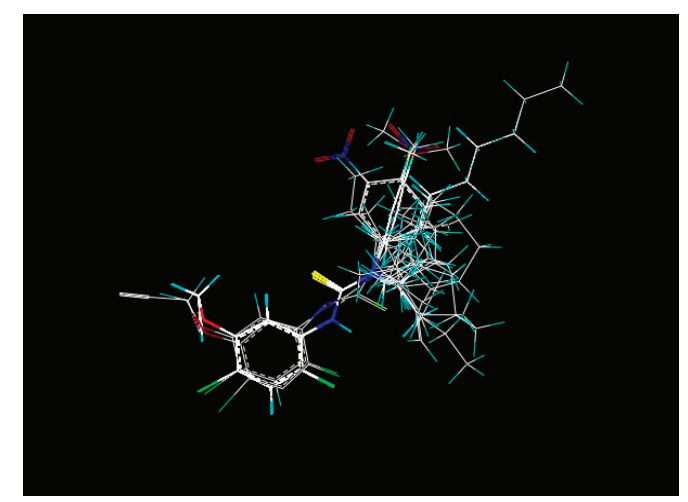

Figure 1. Alignment of the potential energy minimized the Thioureas structures according to a least-squares atom based fit. pre-emergence step. For molecular modeling, Sybyl (Ver. 8.0) software package (Tripos Inc.) was used. ${ }^{13}$ The steric and electrostatic field energies were calculated using $\mathrm{sp}^{3}$ carbon probe atoms with +1 charge. And maximum energy cutoff for the steric and electrostatic energies was $30 \mathrm{kcal} \mathrm{mol}^{-1}$. The grid spacing was $1.0 \sim 3.0 \AA$ in all three dimensions within the defined region. To review the most stable conformer, a random search ${ }^{14}$ was used, and for the energy minimization Tripos force field by Gästier-Hückel charge was performed. ${ }^{15}$ The alignment was carried out by using the SYBYL QSAR rigid body field fit (FF) and atom based fit (AF) command within the SYBYL and using compound $\mathbf{6}$ as a template molecule. ${ }^{16}$ For an example, the AF alignment in three-dimensional space of the potential energy minimized structures and the results of the alignment of all 43 molecules are shown in Figure 1. A training set, composed of 28 of the total 35 data set compounds, was derived from the CoMFA $^{17}$ and CoMSIA ${ }^{18}$ models, and 7 were chosen as the test set. These sets are formed based on the diversity in the structure and the herbicidal activity. The statistical validity of the model was judged by the high values of $\mathrm{q}^{2}$ (or $\mathrm{r}_{\mathrm{cv} \text {. }}^{2}$ ) (more than 0.5 ) and $r^{2}$ ncv. (more than 0.9) with low standard error of estimate (SEE). And additional descriptor such as calculated $\log \mathrm{P}$ was used as independent variable to obtain an improved 3D-QSAR (CoMFA) model. To derive models with a statistically significant correlation, compounds that were derived from correlation between the predictive values (Pred. $\mathrm{pI}_{50}$ ) by the optimized models and the observed values $\left(O b s . \mathrm{pI}_{50}\right)$ were selected as the test set.

PLS and scrambling analysis. To analyze the correlation between descriptors on the structural characteristics of compounds aligned on the three dimensional spaces and the observed herbicidal activity $\left(O b s . \mathrm{pI}_{50}\right)$ according to the alrylamino (R) substituents (hereinafter referred to as the R-substituents) of the Thioureas, PLS (partial least square) analytical method in the SYBYL package was used. ${ }^{19}$ CoMFA models were derived from the combined conditions of the steric field, electrostatic field, and hydrophobic field $(\log \mathrm{P})$ as an additional descriptor. ${ }^{20}$ CoMSIA models by combination of five fields such as the steric filed, the electrostatic field, the hydrophobic field, the H-bond donor and the H-bond acceptor field were derived. ${ }^{17,19}$ To determine the optimized component number, LOO (leave-one-out) validated process was performed and the prediction value $\left(\mathrm{q}^{2}\right.$ or $\mathrm{r}_{\mathrm{cv} \text {. }}^{2}$ ) and component number were explored. The non-validated process was performed to acquire the correlation coefficient $\left(\mathrm{r}^{2}\right.$ ncv. $)$ and 3D-QSAR analysis was applied. From the crossvalidated results, PRESS (predictive residual sum of squares) between the observed values and the predictive values were calculated to determine the predictability of the models. In the statistical values of CoMFA and CoMSIA models, $n$ is the number of compounds and $r$ the correlation coefficient, $\mathrm{r}_{\text {ncv. }}^{2}$. the squared correlation coefficient, $s$ the standard deviation, and $\mathrm{q}^{2}\left(\right.$ or $\mathrm{r}_{\mathrm{cv}}^{2}$ ) the predictive correlation coefficient.

In addition, the progressive scrambling procedure was applied for the evaluation of the sensitivity of the 3D-QSAR models to chance correlations and progressive scrambling analyses (maximum: 8 bins, minimum: 2 bins and critical point: 0.85 ) were performed. ${ }^{21}$ Progressive scrambling with $2 \sim 5$ components of the herbicidal data produces three statistical data, the 
Table 2. Summary of the statistical parameters of 3D-QSAR models with two alignments

\begin{tabular}{|c|c|c|c|c|c|c|c|c|}
\hline \multirow{2}{*}{ Model No. } & \multirow{2}{*}{ Alignments } & \multicolumn{7}{|c|}{ PLS Analyses } \\
\hline & & Grid $(\AA)$ & $\alpha^{a}$ & $\mathrm{NC}$ & $\mathrm{r}_{\mathrm{cv} .}^{2} b$ & $\mathrm{r}_{\text {ncv. }}^{2}{ }^{c}$ & $\mathrm{SE}_{\text {ncv. }}{ }^{d}$ & $\mathrm{~F}^{e}$ \\
\hline CoMFA $1^{f}$ & $\mathrm{AF}$ & 1.0 & - & 4 & 0.531 & 0.931 & 0.120 & 85.582 \\
\hline CoMFA 2 & $\mathrm{FF}$ & 1.5 & - & 4 & 0.444 & 0.881 & 0.165 & 42.370 \\
\hline CoMSIA 1 & $\mathrm{AF}$ & 1.5 & 0.5 & 4 & 0.379 & 0.859 & 0.179 & 34.988 \\
\hline CoMSIA 2 & $\mathrm{FF}$ & 1.5 & 0.4 & 4 & 0.374 & 0.857 & 0.180 & 35.578 \\
\hline
\end{tabular}

Notes: AF: atom based fit; FF: field fit; NC: number of PLS component; ${ }^{a}$ attenuation factor; ${ }^{b}$ cross-validated $\mathrm{r}^{2}$; ${ }^{c}$ non-cross-validated $\mathrm{r}^{2}$; ${ }^{d}$ standard error estimate; ${ }^{e}$ fraction of explained versus unexplained variance; ${ }^{f}$ optimized model.

Table 3. Herbicidal activity $\left(\mathrm{pI}_{50}\right.$ ) of the Thioureas against barnyard grass and predicted activity by optimized CoMFA model 1 for test set

\begin{tabular}{llrrr}
\hline \multirow{2}{*}{ No. } & \multirow{2}{*}{ Substituents (R) } & \multicolumn{3}{c}{$\mathrm{pI}_{50}$} \\
\cline { 3 - 5 } & & Obs. $^{a}$ & Pred. $^{b}$ & $\Delta \mathrm{pI}_{50}{ }^{c}$ \\
\hline $\mathbf{1 1}$ & cy-hexyl & 3.55 & 3.92 & -0.37 \\
$\mathbf{1 5}$ & di-iso-propyl & 4.08 & 3.74 & 0.34 \\
$\mathbf{1 7}$ & di- $n$-butyl & 3.18 & 3.65 & -0.47 \\
$\mathbf{1 9}$ & phenyl & 3.33 & 3.52 & -0.19 \\
$\mathbf{2 0}$ & 4-tolyl & 3.70 & 3.29 & 0.41 \\
$\mathbf{2 9}$ & 3-nitrophenyl & 3.72 & 3.02 & 0.70 \\
$\mathbf{3 5}$ & 3,4-ifluorophenyl & 3.77 & 4.01 & -0.24 \\
\hline
\end{tabular}

${ }^{a}$ Observed herbicidal activity; ${ }^{b}$ predicted activity by the model; ${ }^{c}$ different between observed and predicted activity.

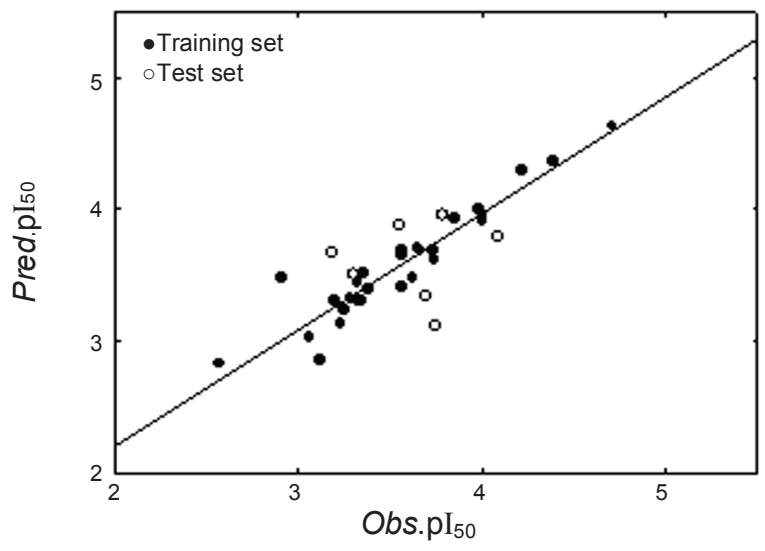

Figure 2. Actual activity $\left(O b s . \mathrm{pI}_{50}\right)$ versus predicted herbicidal activity (Pred.pI ${ }_{50}$ ) for the training and test sets of the optimized CoMFA model 1 (For training set: Pred.pI $\mathrm{I}_{50}=0.939$ Obs. $\mathrm{pI}_{50}+0.218, \mathrm{n}=28$, $\left.\mathrm{s}=0.110, \mathrm{~F}=383.21, \mathrm{r}^{2}=0.936 \& \mathrm{q}^{2}=0.921\right)$.

predictivity $\left(\mathrm{q}^{2}\right)$ of the model, the calculated cross-validated standard error (cSDEP) and the sensitivity to perturbation $\left(\mathrm{d}_{\mathrm{q}}{ }^{2} / \mathrm{dr}^{2} \mathrm{yy}^{\prime}\right)$.

CoMFA contour maps. To analyze the visual structural characteristics of the active sites expected from the optimized CoMFA model 1, the CoMFA characteristics field STDEV* COEFF contour maps from the best predictive PLS analysis and atom based alignment with $\log \mathrm{P}$ as additional descriptor are plotted. We analyzed the CoMFA contour maps on the most herbicidal activity to the EC. The most herbicidal active compound was the allyl substituent $6\left(\mathrm{pI}_{50}=4.71\right)$. On the contour maps, the ratio of favor and disfavor contribution (\%) was $80 \%$ and $20 \%$, respectively.

\section{Results and Discussion}

Optimized CoMFA model. The observed activities (Obs.pI $\left.I_{50}\right)$ of the R-substituents in the Thioureas against the EC and the predicted activities (Pred.pI $\mathrm{I}_{50}$ ) by the optimized CoMFA model 1 and difference values $\left(\Delta \mathrm{pI}_{50}\right)$ between the observed activity and the predicted activity are summarized in Table 1. Also, Table 2 shows the statistical results for the CoMFA and CoMSIA models in several different field combinations based on two alignments (AF and FF) and changes of the gride $(1.5 \sim 3.0 \AA){ }^{22}$ The predictability $\left(\mathrm{r}_{\mathrm{cv}}^{2}\right.$ ) of the CoMSIA models and the CoMFA model 2 were $0.374 \sim 0.444$, while the correlation coefficient $\left(r^{2}\right.$ ncv. $)$ were $0.857 \sim 0.881$ under the AF and the FF alignment condition. In particular, since the CoMSIA models and the CoMFA model 2 were not statistically satisfied the standard $\left(\mathrm{r}_{\text {ncv. }}^{2}>0.9\right.$ and $\left.\mathrm{r}_{\text {nc.. }}^{2}>0.5\right)$ of model with appropriate correlation and predictability, ${ }^{19}$ they were inappropriate models from stability and rational predictability. For the CoMFA model 1, the prediction values $\left(\mathrm{r}_{\mathrm{cv}}^{2}\right)$ was 0.531 , while the relativity value $\left(r^{2}\right.$ ncv. $)$ was 0.931 with a good correlation. Therefore, we selected the CoMFA model 1 as the best model. For the alignment condition, the statistical values of the $\mathrm{AF}$ alignment condition were better statistically suitable than those of the FF alignment condition $(\mathrm{AF}>\mathrm{FF})$. Based on the Table 1, Figure 2 explains the relation between observed activity $\left(O b s . p I_{50}\right)$ of the training set compounds and the predicted activity (Pred.pI $\mathrm{I}_{50}$ ) by the optimized CoMFA 1 model. This linear relation (for the training set: Pred. $\mathrm{pI}_{50}=0.939$ Obs.pI $\mathrm{I}_{50}+0.218, \mathrm{n}=28, \mathrm{~S}=0.110, \mathrm{~F}=$ $383.21, \mathrm{r}^{2}=0.936$ and $\mathrm{q}^{2}=0.921$ ) shows a good correlativity of the CoMFA model 1 but test set compounds were a little bit out of the line.

In order to verify the predictability of the optimized CoMFA model 1, the CoMFA analysis of the test set composed of seven compounds was reported in Table 3. The contribution ratio (\%) of characteristic fields and the errors (Ave. \& PRESS) of training set and test set $(n=7)$ compounds were calculated and summarized in Table 4. According to the data, the correlativity $\left(\mathrm{r}^{2}\right.$ ncv. $)$ of the optimized CoMFA model 1 is good but the predictability ( $\mathrm{r}_{\mathrm{cv}}^{2}$ ) is not good as we expected in the Figure 2 . The field contribution of the CoMFA model 1 in Table 4 is as follows: the steric field $(76.9 \%)>$ the electrostatic field $(20.1 \%)>$ the hydrophobic field (3.0\%). The contribution of the steric field 
Table 4. Summary of field contribution, Ave. and PRESS of 3D-QSAR models

\begin{tabular}{|c|c|c|c|c|c|c|c|}
\hline \multirow{2}{*}{ Model No. } & \multicolumn{3}{|c|}{ Field contribution $(\%)$} & \multicolumn{2}{|c|}{ Training set } & \multicolumn{2}{|c|}{ Test set } \\
\hline & $\mathrm{S}$ & $\mathrm{E}$ & Hy & Ave. & PRESS & Ave. & PRESS \\
\hline CoMFA $1^{a}$ & 76.9 & 20.1 & 3.0 & 0.09 & 0.33 & 0.39 & 1.23 \\
\hline CoMFA 2 & 80.9 & 16.3 & 2.8 & 0.12 & 0.63 & 0.39 & 1.28 \\
\hline CoMSIA 1 & 52.3 & 47.7 & - & 0.12 & 0.75 & 0.37 & 1.33 \\
\hline CoMSIA 2 & 51.2 & 48.8 & - & 0.12 & 0.75 & 0.34 & 1.15 \\
\hline
\end{tabular}

Notes: S: steric; E: electrostatic; Hy: hydrophobic; Ave.: average residual; PRESS: predictive residual sum of squares; ${ }^{a}$ optimized model.

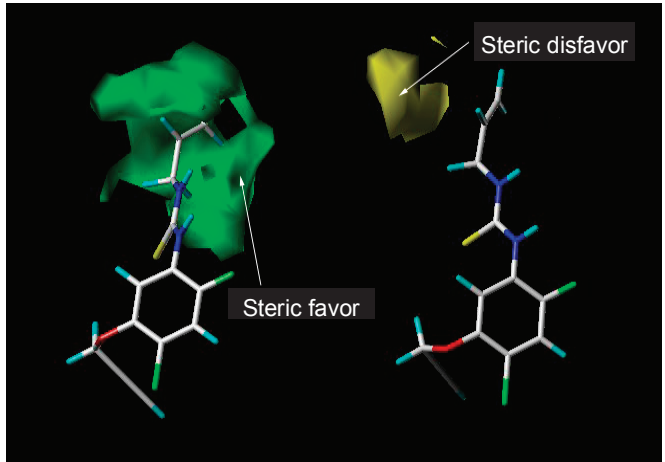

Figure 3. The contour maps for steric field. The most active compound (6) is shown in capped sticks (favor, left: 80\% \& disfavor, right: $20 \%$ ).

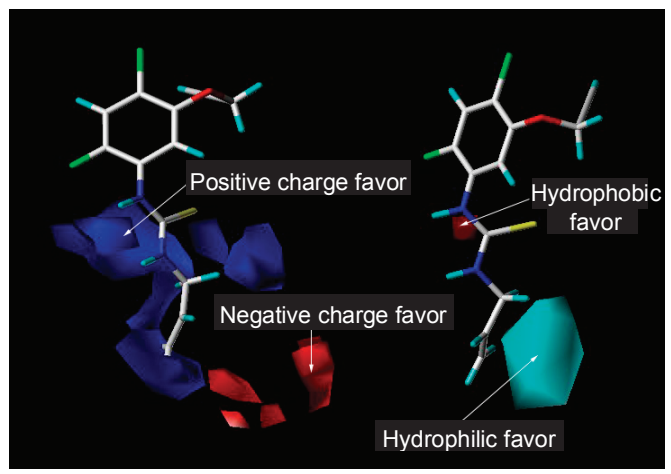

Figure 4. The contour maps for electrostatic field and CoMFA-HINT map for hydrophobic field. The most active compound (6) is shown in capped sticks (hydrophobic favor: $80 \%$ \& hydrophilic favor $20 \%$ ).

Table 5. Model stability test for the optimized CoMFA 1 model by progressive scrambling

\begin{tabular}{cccc}
\hline $\mathrm{NC}^{a}$ & $\mathrm{q}^{2 b}$ & $\operatorname{cSDEP}^{c}$ & $\mathrm{~d}_{\mathrm{q}}{ }^{2} / \mathrm{dr}^{2}{ }_{\mathrm{yy}}{ }^{d}$ \\
\hline 2 & 0.229 & 0.402 & 0.613 \\
3 & 0.339 & 0.380 & 0.665 \\
4 & 0.475 & 0.349 & 1.081 \\
5 & 0.442 & 0.365 & 0.665
\end{tabular}

${ }^{a}$ Number of PLS component; ${ }^{b} \mathrm{q}^{2}=1-(\mathrm{sSDEP})^{2}$, predictivity of the models; ${ }^{c}$ calculated cross-validated standard error; ${ }^{d}$ slope of $\mathrm{q}^{2}$, crossvalidated correlation coefficient.

was three-fold higher than that of the electrostatic field and the hydrophobic field had the least influence. Specifically, this shows that the steric field is the most important factor in the herbicidal activity of the Thioureas against the EC.

Progressive scrambling. To evaluation of the sensitivity of the optimized CoMFA model 1 to chance correlations, the leave-one-out (LOO) cross-validation and progressive scrambling analyses were performed with $3 \sim 5$ components. ${ }^{21}$ In this approach, small random perturbations are introduced into a data set and the statistical results, the perturbation prediction $\left(\mathrm{q}^{2}\right)$, the calculated cross-validated standard error of prediction (cSDEP) as the function of the correlation coefficient between the true values $(\mathrm{y})$ of the dependent variables and the perturbed values ( $y^{\prime}$ ) of the dependent variables, and the slope of $q^{2}$ (crossvalidated correlation coefficient) with respect correlation of the original dependent variables versus the perturbed dependent variables $\left(\mathrm{d}_{\mathrm{q}}{ }^{2^{\prime}} / \mathrm{dr}^{2} \mathrm{yy}^{\prime}\right)$, are summarized in Table 5. Specifically, in case of four components, the sensitivity to the perturbation $\mathrm{d}_{\mathrm{q}}{ }^{2} / \mathrm{dr}^{2} \mathrm{yy}^{\prime}=1.081$ and the prediction $\mathrm{q}^{2}=0.475$ produced by a progressive scrambling analyses were not dependent on chance correlation. Therefore, the data suggested that four components were optimal because a number of PLS components yielding $\mathrm{a} \mathrm{d}_{\mathrm{q}}{ }^{2} / \mathrm{dr}^{2}{ }_{\text {yy' }}$ slope near unity should be optimal. ${ }^{23}$

Analysis of CoMFA contour maps. Figure 3 and 4 show the contour maps of the optimized CoMFA model 1 for the EC. Figure 3 indicates the contour maps of the steric field of the $\mathrm{R}$-substituent in the Thioureas that has the highest relative contribution ratio (\%) to the herbicidal activity. The characteristics of the substituent based on the steric field (S: $76.9 \%$ ) are as follows: a steric favor group (green polyhedra) is on the part close to the nitrogen atom of the R-substituent, and a steric disfavor group (yellow polyhedra) is on the outer side of the $\mathrm{R}$-substituent. Thus, as the size of the R-substituent increases, so does the herbicidal activity of the substituent. Figure 4 shows the contour maps of the electrostatic field (E: 20.1\%) and the hydrophobic field (Hy: 3.0\%). The characteristics of the Rsubstituent based on the electrostatic field are as follows, the terminal part of the R-substituent needs a negative electric charge (red polyhedra) in order to increase the inhibitory activity, whereas the beginning part of the R-substituent requires a positive charge (blue polyhedra) with a relatively wide range except the phenyl ring. It is expected that in the contour maps of the hydrophobic field, the activity is improved as the end part of the R-substituent has bigger hydrophobicity.

Acknowledgments. This work was supported by the National Research Foundation (NRF) grant (No. 2010-0062913) funded by the Korea Government (MEST). 


\section{References}

1. (a) Fujita, T.; Nakayama, A. In Peroxidizing Herbicides: Structureactivity relationship and molecular design of peroxidizing herbicides with cyclic imide structures and their relatives; Boger, P., Wakabayashi, K. Eds.; Springer-Verlag Berline: Heidelberg, Germany, 1999; Ch. 4. (b) Wan, J.; Zhang, L.; Yang, G.; Zhan, C. G. J. Chem. Inf. Comput. Sci. 2004, 44, 2099. (c) Hiraki, M.; Ohki, S.; Sato, Y.; Jablonkai, I.; Boger, P.; Wakabayashi, K. Pestic. Biochem. Physiol. 2001, 70, 159. (d) Murata, S.; Kimura, Y.; Mabuchi, T.; Miura, Y. J. Pestic. Sci. 2004, 29, 195. (e) Theodoridis, G.; Bahr, J. T.; Hotzman, F. W.; Sehgel, S.; Suarez, D. Crop. Protection 2000, 19, 533.

2. (a) Uraguchi, R.; Sato, Y.; Nakayama, A.; Sukekaya, M.; Iwataki, I.; Boger, P.; Wakaba- yashi, K. J . Pesticide Sci. 1997, 22, 314. (b) Selby, T. P.; Armel, G. R.; Hughes, K. A.; Chen, Y.; Xu, M.; Stevenson, T. M.; Kamireddy, B. ; Mauvais, P. A. Abstracts of Papers, 236th ACS National Meeting, Philadelphia, PA, United States, August 17-21, 2008.

3. (a) Nandihalli, U. B.; Duke, M. Y.; Duke, S. O. Pestic. Biochem. Physiol. 1992, 43, 193. (b) Luo, Y. P. ; Jiang, L. L.; Wang, G. D.; Chen, Q.; Yang, G. F. J. Agri. Food Chem. 2008, 56, 2118.

4. Matsumoto, H. Herbicide Modes of Action, Recent Progress and New Challenges Proceeding of the 17th Asian-Pacific Weed Science Society Conference; 2000; 39.

5. Boger, P., Wakabayashi, K., Eds.; In Peroxidizing Herbicides; Springer-Verlag Berline: Heidelberg, Germany, 1991; 44.

6. Scalla, R.; Matringe, M.; Camadro, J. M.; Labbe, P. Z. Naturforsch. 1990, 45c, 503.

7. (a) Sung, N. D.; Yoon, T. Y.; Chung, H. S. Korean J. Pestic. Sci. 2005, 9, 146. (b) Soung, M. G.; Lee, Y. J.; Sung, N. D. Bull. Korean Chem. Soc. 2009, 30, 613.

8. (a) Sung, N. D.; Kang, E. K.; Song, J. H.; Jung, H. S. Korean J. Pestic. Sci. 2005, 9, 199. (b) Sung, N. D.; Kang, E. K.; Song, J.
H.; Jung, H. S. J. Korean Soc. Appl. Biol. Chem. 2005, 48, 394.

9. (a) Sung, N. D.; Jung, K. S.; Jung, H. S.; Chung, Y. H. Korean J. Pestic. Sci. 2006, 10, 1. (b) Chung, K. S.; Jang, S. C.; Choi, K. S.; Sung, N. D.; J. Korean Soc. Appl. Biol. Chem. 2006, 49, 238.

10. Akamatsu, M. Curr. Topics Med. Chem. 2002, 2, 1381.

11. (a) Cramer, R. D.; Patterson, D. E.; Bunce, J. D. J. Am. Chem. Soc. 1988, 110, 5959. (b) Cramer, R. D., III.; Wold, S. CoMFA US Patent application No. 237491, August 1988.

12. (a) Park, K. Y.; Song, J. H.; Jeon, D. J.; Soung, M. G.; Sung, N. D. Korean J. Pestic. Sci. 2008, 12, 103. (b) Soung, M. G.; Park, K. Y.; Song, J. H.; Sung, N. D. J. Korean Soc. Appl. Biol. Chem. 2008, 51, 219.

13. Tripos, Sybyl. Molecular modeling and QSAR software on CDRom (Ver. 8.0), Theory and manual, Triopos Associates, Inc., Suite 303, St. Louis, MO.

14. Gilbert, K. M.; Venanzi, C. A. J. Comput. Aided-Mol. Des. 2006, 20, 209.

15. Clark, M.; Cramer, R. D. III.; Van Opdenbosch, N. J. Comput. Chem. 1989, 10, 982.

16. Klebe, G. In 3D-QSAR Drug Design, Theory, Methods and Applications: Structural Alignment of Molecules; Kubinyi, H., Ed.; 1993; p 173.

17. Cramer, R. D.; Bunce, J. D.; Patterson, D. E. Quant. Struct. Act. Relat. 1988, 7, 18.

18. Klebe, G.; Abraham, U.; Mietzner, T. J. Med. Chem. 1994, 37, 4130.

19. Wold, S.; Ruhe, A.; Wold, H. ; Dunn, W. J. SIAM J. Sci. Stat. Comp. 1984, 5, 735 .

20. (a) Gaillard, P.; Carrupt, P.A.; Testa, B.; Boudon, A. J. Comput. -Aided. Mol. Des. 1994, 8, 83. (b) Wade, R. C. Structure 1977, 5, 1139.

21. Clark, R. D.; Fox, P. C. J. Comput. -Aided. Mol. Des. 2004, 18, 563.

22. Klebe, G.; Abraham, U. J. Comput. -Aided. Mol. Des. 1999, $13,1$.

23. (a) Clark, R. D.; Fox, P. C. J. Comput.-Aided. Mol. Des. 2003, 17, 1.; Clark, R. D.; Sprous, D. G.; Leonard, J. M. Validating Models based on Large Data Sets; Prous Science: Barcelona, 2001; 475. 